\title{
Structural Assessment of Scars Using Optical Techniques
}

L. van Haasterecht, Paul P. M. van Zuijlen, and ML. Groot

\section{Contents}

19.1 Introduction - 170

19.1.1 Volumetric Analyses - 170

19.1.2 Surface Topology -170

19.1.3 Thickness -171

19.2 Experimental Techniques - 171

19.2.1 Optical Coherence Tomography - 172

19.2.2 Confocal Microscopy - 172

19.2.3 Nonlinear Optical Microscopy - 173

19.3 Future Perspectives $\mathbf{- 1 7 5}$

References - 176 


\subsection{Introduction}

The skin, the largest organ of the body, consists of an epidermal layer and a dermal layer underneath. The thin epidermal layer functions as a barrier to the outer world. The dermis is much thicker, and its structural organization is responsible for the functional qualities of the human skin. It consists for almost $80 \%$ of collagen, elastin, and different types of cells, which render the skin strong and at the same time remarkably pliable. In scar tissue, visual aspects (discoloration and texture [1]), functional aspects (stiffness [1] and moisture retention [2]), and sensory functions [3] of the skin differ significantly from those of healthy skin.

In clinical practice, objective assessment of cutaneous scars remains a challenging task. The use of scar assessment tools has increased significantly in the last decade. The clinical follow-up of scar treatment necessitates precise measurement of thickness, surface area, and relief. Further, a better visualization of the precise 3D microstructural organization is crucial in understanding the etiology of pathological scarring. As most aspects of clinical scar assessment scales are based on the visual appearance, the potential for optical techniques as an objective assessment tool is high. With a so-called optical biopsy, it is possible to assess the microstructure below the surface of the skin with optical methods, negating invasive physical biopsies. Furthermore, a range of extensions for these techniques allow simultaneous structural, mechanical, and even chemical characterization.

This chapter aims to describe current progress in objective imaging techniques used in the clinical followup of scar progression on the one hand and some of the most powerful techniques for basic structural research of scar microstructures on the other hand. For the sake of brevity, we limit our discussion to techniques and devices that have been shown to be valid and reliable. For a complete overview of available devices, we refer the reader to an extensive review by Lee et al. [4].

\subsubsection{Volumetric Analyses}

The number of devices that measure scar volume has seen a rapid growth in the last decade, partly advanced by increased attention to wound and burn research.

Volumetric aspects of scars discussed here are surface area, thickness, and surface topology. Although there exists significant overlap between these aspects and some devices measure multiple aspects (surface roughness can be considered as fluctuations in scar height/thickness; newer analysis methods of surface area are often intrinsically three dimensional and therefore include information on surface topology), we dis- cuss them separately as the reliability (the consistency of the measurements) and validity (whether the device measures what it intends to measure) of individual aspects of a device aren't necessarily similar.

Volumetric assessment has gone from manual paper tracing to high-tech 3D cameras.

Although manual tracing of scar surface area is reliable in the case of linear postoperative scars [5], for scars with irregular boundaries, the reliability decreases with scar size [6]. The LifeViz 3D camera (Quantificare SA, Valbonne, France) has been extensively tested in both the surface area and volumetric analysis mode by Stekelenburg et al., who reported it to be a reliable and valid tool $[7,8]$. The LifeViz $3 \mathrm{D}$ works by comparing two photos from different angles, from a predefined distance. The Vectra 3D is a speckle pattern-based system that utilizes six cameras to construct a $3 \mathrm{D}$ image. Its interrater reliability was found to be excellent in a trial on raised scars [9]. A device with a similar working mechanism, MAVIS III (Photometrix, Pontypridd, United Kingdom), was assessed in a study on burn scars. The authors note that poorly delineated scars on curved surfaces pose a challenge in the boundary selection and subsequent analysis [10].

Rashaan et al. tested another stereophotogrammetry device, the Artec MHT (Artec 3D, San Diego, CA, USA), and found it to be highly reliable and valid in burn scars [11].

Other strategies that include laser scanning cameras and structured light scanners need additional clinical evaluation.

\subsubsection{Surface Topology}

Roughness, or surface irregularity, has a significant impact on a patient's perception of scars [12]. Furthermore, the predictive validity of long-term scar quality was found to be significantly influenced by the "relief" item on the POSAS observer scale [13] (see - Chap. 16 for more on scar assessment scales). Most scar assessment scales include such a texture item, and even though subjective analysis isn't necessarily inferior to objective analysis, quantification of these aspect remains challenging.

Early attempts at quantitative evaluation of roughness entailed the casting of a positive or negative mold of the scar surface and its subsequent optical or mechanical analysis [14]. This process, although accurate, is overly cumbersome and time-consuming. Current contactless techniques use a combination of illumination and analysis of disturbance patterns. The most frequently used device, the Phaseshift Rapid In Vivo Measurement of the Skin (PRIMOS, Canfield Scienitifc, Inc., Parsippany, USA), has been shown to reliably mea- 
sure scar topology $[15,16]$. PRIMOS projects patterns of light onto the skin surface. Fluctuations in the surface profile cause disruptions in these patterns that are subsequently analyzed.

Antera 3D (Miravex, Dublin, Ireland) is based on photometric stereo, in which light from different angles produces shade based on surface topology. The reflected light is analyzed to form a surface reconstruction. Similarly, LifeViz Micro (Quantificare SA, Valbonne, France) is one of the devices in the LifeViz series mentioned earlier. Although they have been described in the context of clinical scar assessment $[17,18]$, their clinimetric properties have not been analyzed for the surface roughness aspect.

The Visioscan VC 98 uses software analysis of grayscale values in UVA-illuminated photographs (Visioscan VC 98). Reliability in healthy skin relies heavily on the chosen outcome measure of the "surface evaluation of living skin" method [16].

The analysis of relief can be considered a structural analysis of scar volume. Indeed, most devices offer some volumetric analysis. Furthermore, the Antera 3D offers additional colorimetric analysis. The abovementioned devices show great heterogeneity in measured parameters, resolution, and field of view, and thus, it is difficult to make a qualitative comparison. In the future, further cross-pollination between techniques can be expected. Askaruly et al. showed the potential of optical coherence tomography (OCT) to measure relief [19]. Although their study was not on scar tissue, the possibility of combining 3D surface analysis with structural analysis looks promising.

\subsubsection{Thickness}

Follow-up of scar thickness is especially important in both hypertrophic and keloid scars, where the aberrant deposition of ECM proteins leads to significant expansion. Therapies are mostly focused on reducing the scar's prominence over the surrounding tissue, and correctly analyzing the thickness of cutaneous scar is paramount.

Although not an optical technique, we discuss highfrequency ultrasound here as it's by far the most commonly used technique. Its inferior resolution compared to optical equivalents is mitigated by its superior penetration depth allowing thickness analysis, even in severe scar thickening. The working mechanism is based on reflection of sound waves of structures with different acoustic impedances and the analysis of the reflection time to determine the depth of the structure. The penetration depth ranges from the upper dermal layers to full-thickness skin and subcutaneous structures, depending on the employed frequency.

Numerous ultrasound systems exist that are marketed for dermatological applications; we succinctly limit our discussion to systems that have been applied to scars. The frequency used by these systems ranges from 5 to $50 \mathrm{MHz}$ and forms the most important limitation in scars, as this limits the penetration depth. For example, the most frequently described device, the Dermascan C (Cortex Technology ApS), utilizes a frequency of $20 \mathrm{MHz}$ which has a penetration depth described as inadequate in up to a third of burn scars in a study by Gankande et al. [20] (using the DermaLab Combo).

Reliability of these instruments has been tested in several studies and shows excellent inter-observer [20 $23]$ and intra-observer reliability [20, 21, 23-25].

From a validity standpoint, it is of interest to note that a golden standard does not currently exist. Comparison with scar assessment scales has yielded inconsistent results, possibly due to heterogeneity in scar scales and ultrasound frequency $[23,26]$. Histology has several drawbacks that include deformation during the biopsy process dependent on resting skin tension, manipulation during fixation, and slicing [27]. Agabalyan et al. found poor correlation between $20 \mathrm{Mhz}$ ultrasound analysis and histological analysis of skin graft scars in a small group of patients [26]. Andrew et al. found biopsies to be significantly thicker after excision, advising the use of pinning during formaldehyde fixation to mitigate this effect [27]. Li et al. compared ultrasound measurements with the use of a micrometer [24], theoretically bypassing the negative effects of sample preparation for histology. Although they achieve a high correlation, sonographic analysis was performed on porcine excised tissue in which it is conceivably easier to recognize the skin's boundaries.

Scar height can be measured using volumetric analyses like the 3D camera LifeViz 3D (Quantificare SA, Valbonne, France), although studies comparing this device with ultrasound measurements have favored the latter $[23,28]$. It seems that the reliability is suboptimal due to the device having difficulty scanning over curved surfaces.

\subsection{Experimental Techniques}

Scarring involves the aberrant deposition of matrix molecules. The collagen content of scars, especially, differs from healthy skin in terms of amount, type, and, importantly, organization. In the past decades, quantification of the extracellular matrix (ECM) organization was mostly limited to two-dimensional images from fixated and stained tissue. Depending on the orientation of histological slices relative to the skin surface, analysis of orientation will yield different results. For example, Verhaegen et al. found significantly different supramolecular organization depending on which cross section was imaged (e.g., perpendicular to the surface or in the 
parallel plain) [29]. Gaining a better three-dimensional perspective of ECM organization is paramount in elucidating the etiology of pathological scars.

As the goal is not only to assess scar tissue based on texture, volume, and color but also to perform microstructural analysis, more advanced microscopic tools are required. An ideal optical scar assessment technique would have a resolution with which microstructural changes can be clearly identified, even in the deeper parts of the dermis. However, in optics, there exists a common trade-off between spatial resolution and penetration depth, and the marked thickening of hypertrophic and keloids scar complicates this trade-off. In this sense, a "perfect technique" does not exist. A technique's suitability depends merely on whether the application matches its strength. In the following, we will discuss several microscopic techniques and their applications in scar assessment and research.

\subsubsection{Optical Coherence Tomography}

Colloquially known as "Ultrasound based on light," OCT is a promising technique based on low-coherence interferometry. In short, light with a broad bandwidth is split into a sample arm and a reference arm. The interference pattern that arises from the combination of the reference arm and the sample arm, containing reflected light from different depth layers of the sample, creates a depth profile of the sample. Combining a lateral series of these profiles creates a cross-sectional image (B scan) or a three-dimensional reconstruction when a stack of $\mathrm{B}$ scans is combined. Scanning approaches with full-field illumination allow the acquisition of stacks of "en face" scans. Typically, penetration depths of 1-2 mm can be reached, at a resolution of 4-10 $\mu \mathrm{m}$ [30].

The capability of OCT to create high-resolution tomography first piqued the interest of the ophthalmological community, where it is now a staple technique in retinal assessment. Naturally, attributes like fast acquisition time, high-resolution images, noninvasiveness, and multifaceted structural assessment make OCT an interesting technique for dermatology. Moreover, OCT devices are often manageable handheld scanners, increasing their usability in the clinic.

Polarization-sensitive OCT (PS-OCT) has been described as a quantification tool for collagen density and alignment by means of tissue birefringence [31]. Jaspers et al. compared burn scars and healthy skin using a volume-based birefringence method, which showed strong correlation to histologically determined collagen content [32]. By applying speckle decorrelation, Liew et al. visualized the microvasculature of burn scars in three dimensions [33]. A similar strategy was used in the clinical follow-up of burn scars undergoing laser ablation [34], allowing the authors to distinguish mature and immature scars [35]. Comparable strategies have been applied to lymph vessels [36]. Combined vascularity, lymph vessels, and birefringence analyses were used by Park et al. to longitudinally follow wound healing in a mouse model, showing angiogenetic changes and realignment of birefringent signals [37].

Simultaneous mechanical testing allows the correlation of local structural changes to variations in stiffness. Es'haghian et al. showed that combining a handheld OCT probe with a mechanical loading device permits concurrent imaging of the in vivo scars and stiffness mapping [38, 39]. Vibrational perturbation of the skin and the analysis of resonance frequencies while imaging the layers of the skin have also received some attention recently for bulk measurements of mechanical properties $[40,41]$.

OCT has been described as a tool for measuring thickness of individual skin layers. Because the limited penetration depth makes full thickness imaging impossible, its application is limited to the epidermal thickness $[42,43]$.

\subsubsection{Confocal Microscopy}

Higher-resolution images to assess structures with submicron resolution in $3 \mathrm{D}$ can be obtained using various microscopic techniques. In confocal fluorescence microscopy, the addition of a set of pinholes to a traditional fluorescence microscope allows for the selection and detection of a small focal volume. Selective labeling with a fluorophore allows for the imaging of a particular substance of interest by excitation of the fluorophore by a lamp or laser and detection of the fluorescence emission signals. Images are obtained by scanning the laser or the sample, yielding images with a resolution of $0.4 \lambda / \mathrm{NA}$ where $\lambda$ is the wavelenght of the emitted light and NA is the numerical aperture of the objective, which is an improvement of $\sim 30 \%$ compared to traditional fluorescence microscopy. Another advantage over widefield microscopy is that the labeling with fluorophores can be sparser, allowing for a fairer comparison of relative quantities of structures.

Confocal microscopy has been used to determine the ratios of collagen of different scar types [44], cellsignaling molecules [45] or innervation [46] of different scar types and as an ex vivo tool in evaluation of microneedling penetration depth [47]. Furthermore, the superior resolution allows for quantitative collagen orientation analysis $[29,48]$. Devices like the VivaScope (MAVIG, Munich, Germany) [49] are based on reflectance confocal microscopy (RCM), in which backscattered light from unlabeled structures is analyzed, based on changes in refractive index. The in vivo applications of RCM lie predominantly in the diagnosis of various 
skin cancers [50], as its penetration depth is limited to the epidermis and upper part of the dermis. However, attempts to analyze collagen production after microneedling of acne scars have been described in vivo with a combination of dermoscopy and RCM [51]. The limited penetration depth seems a significant hurdle in scar research; however, combining the device with an OCT arm allowed Iftimia et al. to analyze both structural and birefringence properties in burn scars [52].

\subsubsection{Nonlinear Optical Microscopy}

\subsubsection{Introduction}

As discussed above, confocal microscopy allows for three-dimensional imaging; however in this technique, samples require specific staining and sometimes also fixation and slicing, which may affect ECM structure and organization. Nonlinear optical (NLO) microscopy involves label-free imaging of unfixed tissue in three dimensions and has strong potential in the visualization of the scar microstructure. Here, we discuss several types of NLO microscopy, namely, higher harmonic generation microscopy (HHG) and multiphoton excited autofluorescence (MEA), and explain their strengths in label-free imaging of scars.

Nonlinear microscopy can be described as the conversion of multiple photons into a single photon, based on the specific nonlinear behavior of certain molecules or structures. This process occurs only when the intensity of the incident light is sufficiently high. To keep the average laser power on the sample sufficiently low, short-pulsed lasers are used (generally femtosecond lasers) [53].

MEA involves the absorption of two or more photons by an intrinsic fluorophore and the recording of the subsequent fluorescence emission, which is then at a wavelength shorter than of the excitation light. Alternatively, external fluorophores can be applied to the sample to label specific structures. In contrast to single-photon fluorescence, excitation wavelengths typically lie in the infrared region, allowing deeper tissue penetration of up to $1.6 \mathrm{~mm}$ [54].

Comparably, in the HHG processes, two or three photons are converted into a single photon. No energy transfer takes place however, and the emitted photons have exactly half (second harmonic generation, SHG) or a third (third harmonic generation, THG) of the wavelength of the incident photons and exactly double or triple the energy, respectively. This allows the detection of the specific signal using narrow-banded filters, improving contrast between structures. Simultaneous registration of these signals using one system allows for label-free visualization of the most important structural proteins of the ECM.

\subsubsection{Two-Photon Excited Autofluorescence (2PEF)}

MEP involves two or three photons being absorbed by a fluorophore and the subsequent emission of a single photon of a shorter wavelength. Two-photon excited autofluorescence (2PEF) has been described extensively in skin research.

$2 \mathrm{PEF}$ is applicable to fluorophores used in singlephoton fluorescence. As this is a fundamentally different process, excitation wavelengths will not equate to exactly double the wavelength of the single-photon excitation. The emission spectrum however will be identical. Fluorophores naturally present in biological tissue have well-described excitation and emission spectra, and extrapolation to the two-photon excitation wavelength is usually warranted. Although penetration depths more than one millimeter have been described in the context of brain tissue [54], the density of the skin and the relevant excitation wavelengths do not permit such deep penetration. Imaging systems are conventionally limited to several hundred micrometers in depth.

Both collagen and elastin, the most important structural proteins of the extracellular matrix (ECM), have convenient 2PEF profiles [55]. On the cellular level, the abundantly present cytoplasmic metabolites NADH and FAD are prolific emitters in their reduced and oxidized states, respectively. An example of 2PEF imaging of dermal fibroblasts can be seen in • Fig. 19.1.

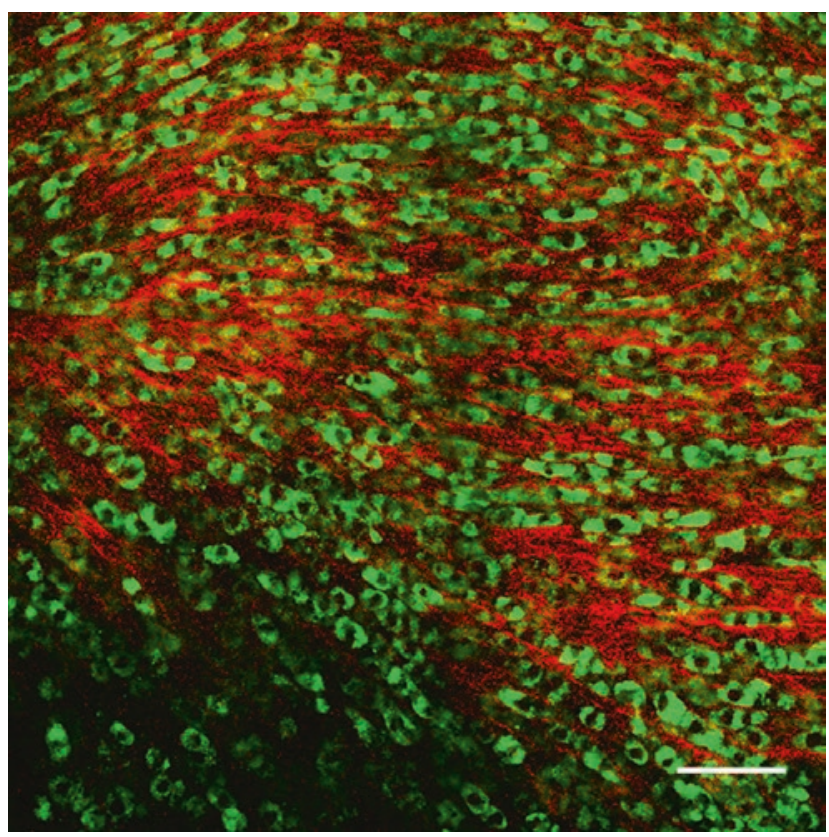

- Fig. 19.1 Combined two-photon excited fluorescence image (2PEF, green) and second harmonic generation image (SHG, red) of cultured human dermal cells and their derived collagen matrix, respectively. Collagen fibers induce a bright SHG signal, whereas intrinsic fluorophores in the cytoplasm of the dermal cells outline the cells. In vitro images were acquired using a previously described setup [87]. Scale bar: $500 \mu \mathrm{m}$ 
Simultaneous recording allows a redox ratio to be calculated. The feasibility of this strategy was shown in an in vivo mouse model by Quinn et al. [56], visualizing metabolic changes during wound healing. Another in vivo study used the same concept to investigate the depth-dependent metabolism of keratinocytes [57]. Melanin content has been used as an in vivo marker for several dermatological disorders [58].

\subsubsection{Second Harmonic Generation (SHG) Microscopy}

The introduction of SHG microscopy has sparked a slew of research in dermatology. Also known as frequency doubling, it involves the interaction of two photons of the same wavelength with a nonlinear material and the subsequent creation of a single photon with double the frequency and energy. The ability of a material for second harmonic generation, described by the nonlinear susceptibility coefficient $\chi(2)$, depends on the non-centrosymmetric structure. Biologically significant harmonophores include collagen [59], myosin [60], and microtubules [61].

In the case of fibrillar collagen, the repeating dipoles of peptide bonds and the structural alignment on all levels of the molecular and supramolecular structure (parallel peptide bonds are assembled into parallel fibrils; fibrils are aligned into fibers) result in coherent amplification of the emitted second harmonic signal refs. This also means that the structural alignment of collagen fibers influences the SHG signal intensity [62]. As this is not the case for non-fibrillar collagen types, SHG microscopy is specific for type I and II collagens and forms a highly specific tool for 3D imaging of unprocessed skin samples as showed in $\bullet$ Fig. 19.2 Abnormal ratios of collagen I:III have been described in multiple fibrotic processes [63]. Although collagen type III, often deposited alongside type I, is a fibrillar collagen, it produces only weak SHG signals [64]. As the SHG signal is dependent on phase-matching conditions, most of the signal forwardly propagated. The thin scattered fibers of collagen type III and their associated reduction in phase matching appear to be distinguishable from type I by comparing forward- and backward-scattered SHG images $[65,66]$. The opacity of collagenous tissues however makes the detection of this signal impractical, rendering this technique possible only in thin specimens.

Multiple strategies to distinguish scar types using SHG images have been described. Most of these focus on software-based analysis of collagen fiber orientation and density [67-69]. Matrix organization and orientation have been described as abnormal in scars [29]. Nonsoftware-based approaches rely on the dependence of the nonlinear susceptibility of collagen on the polarization of the incident light. Polarization-sensitive SHG (PS-SHG) probes the organization of collagen fibers by sequentially rotating the polarization relative to the optical axis and probing the SHG response. By analyzing the SHG intensity at different angles of polarization, orientation vectors can be reconstructed [70, 71]. Note that because of the strong dependence of SHG on polarization, software-based analyses should be performed on images from circularly polarized light sources.

Using unstained tissue allows simultaneous biomechanical testing during imaging. For example, Rosin et al. assessed the scarring capacity of healing splitthickness skin grafts by microstructural SHG imaging of collagen and biaxial stretch testing [72]. The authors correlate the loss of waviness patterns to increased stiffness.

The reduced phototoxicity (as no external fluorophores are used) from these optical processes allows in vitro and even in vivo imaging, for example, skin equivalents [73], lab-grown wound models [74], and antifibrotic treatments [75].

Several research groups have made attempts to include SHG in clinical decision-making. Predominately applied to neoplastic lesions, consecutive follow-up of

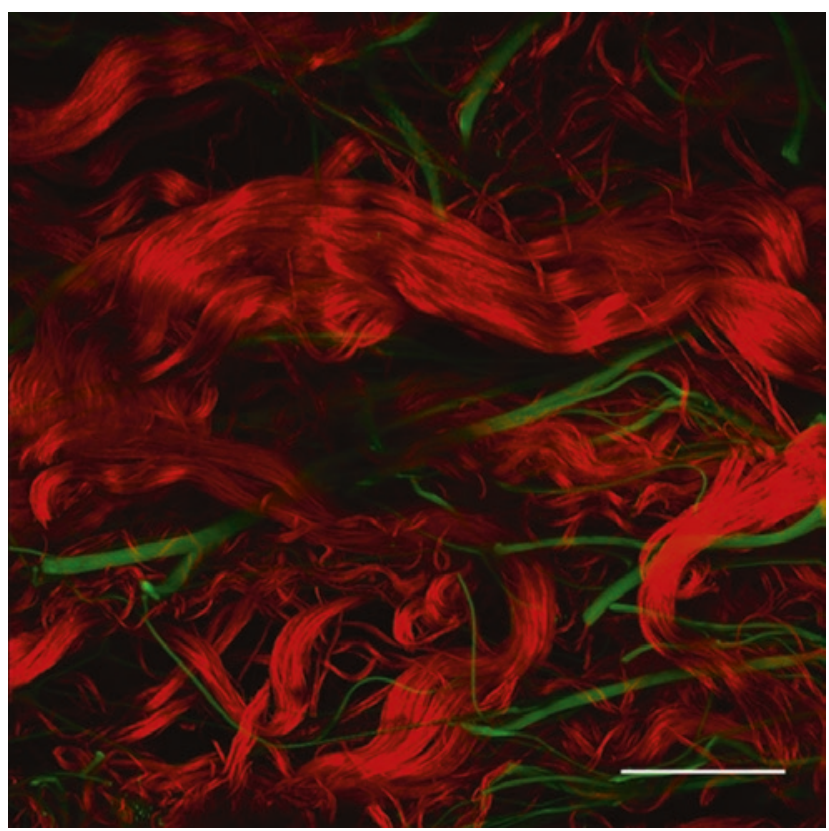

- Fig. 19.2 Combined 2PEF (green) and SHG (red) image of elastin and collagen fibers, respectively. Unprocessed ex vivo human skin, specifically the reticular dermis, was imaged using the setup described in [87]. A stack of 35 images was acquired in the Z-axis. Image processing in Image $J$ resulted in the $3 \mathrm{D}$ projection seen here [88]. Scale bar: $500 \mu \mathrm{m}$ 
scars and topical therapies has also been described. Commercial translation has already resulted in multiple devices marketed for in vivo dermatological applications. The Dermaspect (JenLab GmbH, Berlin, Germany) device entails a multimodal (2PEF, CARS, SHG) microscope with an adjustable scan head for in vivo skin imaging [76]. Innovations in laser technology allow for increasingly smaller and cheaper devices [77, 78].

\subsubsection{Third Harmonic Generation (THG) Microscopy}

The propensity of a material for third harmonic generation depends not only on its nonlinear susceptibility coefficient $\chi(3)$ but also on whether partial phase matching can be achieved by a small inhomogeneity at the focus, making THG an interface-sensitive technique [79]. THG is less specific than SHG, increasing the number of possible applications. By setting the focal volume of the incident laser beam to several times the size of a typical tissue structure, a geometry can be created where the phase-matching conditions enable efficient THG. As the THG contrast comes from inhomogeneities and tissue interfaces with a high $\chi(3)$, cell membranes and cell nuclei are clearly displayed with THG microscopy, since they contain lipids which are known to have a high $\chi(3)$ [80]. Not surprisingly, the multilayered structure of the epidermis provides excellent contrast. - Figure 19.3 shows a THG cross section of the epidermis in a drug penetration experiment. To illustrate the potential in dermatology, THG was shown to be a viable imaging technique for label-free tracking of melanoma cells [81]. The THG contrast from melanin and structural features allowed differentiation of skin cancers [82].

In the context of scar tissue, the surface of elastin fibers is an adequate THG emitter [83]; thus, combining SHG and THG potentially allows for three-dimensional imaging of unstained, unfixed, and unsliced tissue.
Although an analysis of scar tissue has not been described yet, THG has strong potential as a label-free imaging technique because of reduced phototoxicity. Indeed, several articles describe the in vivo "optical biopsy" technique in the skin $[78,84]$.

\subsubsection{Coherent Anti-Stokes Raman Spectroscopy (CARS)}

The structural information provided by SHG and THG can be supplemented by chemical information using another nonlinear process, Coherent anti-Stokes Raman Spectroscopy (CARS). Here, two lasers with different frequencies are focused on the sample, to probe the vibrational fingerprint of the molecules in the focal point. The $\mathrm{CH} 2$ content of lipids provides excellent contrast when imaging phospholipid-rich cell membranes and adipocytes. The $\mathrm{OH}$ signal from water molecules makes this technique especially applicable for research into transepidermal water loss $[76,85]$.

\subsection{Future Perspectives}

The enormous increase in available devices for volumetric analysis is indicative for the increased attention for quantification tools in scar research. Technological advances and the need for point of care solutions have already resulted in smaller and manageable devices suitable for bedside use. This trend can be expected to continue with smartphone-based solutions already being investigated in wound evaluation [86]. Both researcher and clinicians should however be mindful of the fact that most devices are not adequately tested on their clinimetric properties.

This trend is also applicable to experimental techniques. As image acquisition times decrease and devices get smaller, we can expect integration of different imaging techniques, each bringing their own strengths in elucidating the multifaceted issue of pathological scarring.

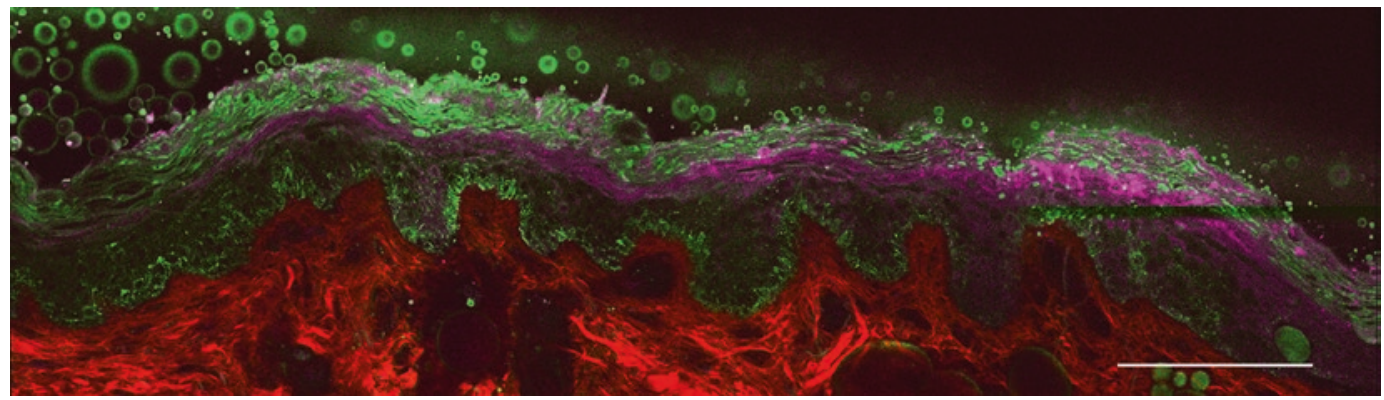

- Fig. 19.3 Nonlinear microscopy image of a cross section of ex vivo skin. The THG signal (green) depicts the layers of the epidermis; collagen fibers in the papillary dermis give rise to the SHG sig- nal in red. In this experiment, fluorescently labeled (magenta) hyaluronic acid $(250 \mathrm{kDa})$ was applied to the skin's surface to quantify epidermal skin penetration. Scale bar: $500 \mu \mathrm{m}$ 


\section{Take Home Messages}

- Technological advances will increase the number of devices for scar assessment, while reducing their size.

- The integration of multiple techniques has the potential to improve scar assessment.

- For most devices, the clinimetric properties are insufficiently determined.

- SHG microscopy is a valuable tool for experimental research into scar pathophysiology and potential therapeutics.

\section{References}

1. Spronk I, Polinder S, Haagsma J, Nieuwenhuis M, Pijpe A, Van der Vlies C, et al. Patient-reported scar quality of adults after burn injuries: a five-year multicenter follow-up study. Wound Repair Regen. 2019;27(4):406-14.

2. Gardien KLM, Baas DC, de Vet HCW, Middelkoop E. Transepidermal water loss measured with the Tewameter TM300 in burn scars. Burns 2016; 42 (7), 1455-62.

3. Brown BC, Mckenna SP, Siddhi K, Mcgrouther DA, Bayat A. The hidden cost of skin scars: quality of life after skin scarring. J Plast Reconstr Aesthet Surg. 2008;61:1049-58.

4. Lee KC, Dretzke J, Grover L, Logan A, Moiemen N. A systematic review of objective burn scar measurements. Burns Trauma 2016;4:14

5. Feldstein S, Wilken R, Wang JZ, Taylor SL, Eisen DB. Development and initial validation of the trace-to-tape method: an objective outcome measure for linear postoperative scars. Br J Dermatol. 2019;181(3):633-4.

6. Van Zuijlen PPM, Angeles AP, Suijker MH, Kreis RW, Middelkoop E. Reliability and accuracy of techniques for surface area measurements of wounds and scars. Int $\mathbf{J}$ Low Extrem Wounds. 2004;3(1):7-11.

7. Stekelenburg CM, Van Der Wal MBA, Knol DL, De Vet HCW, Van Zuijlen PPM. Three-dimensional digital stereophotogrammetry: a reliable and valid technique for measuring scar surface area. Plast Reconstr Surg. 2013;132(1):204-11.

8. Stekelenburg CM, Jaspers MEH, Niessen FB, Knol DL, Van Der Wal MBA, De Vet HCW, et al. In a clinimetric analysis, 3D stereophotogrammetry was found to be reliable and valid for measuring scar volume in clinical research. J Clin Epidemiol. 2015;68(7):782-7.

9. Verhiel SHWL, Piatkowski de Grzymala AA, Van den Kerckhove E, Colla C, van der Hulst RRWJ. Three-dimensional imaging for volume measurement of hypertrophic and keloid scars, reliability of a previously validated simplified technique in clinical setting. Skin Res Technol. 2016;22(4):513-8.

10. Su S, Sinha S, Gabriel V. Evaluating accuracy and reliability of active stereophotogrammetry using MAVIS III Wound Camera for three-dimensional assessment of hypertrophic scars. Burns. 2017;43(6):1263-70.

11. Rashaan ZM, Stekelenburg CM, van der Wal MBA, Euser AM, Hagendoorn BJM, van Zuijlen PPM, et al. Three-dimensional imaging: a novel, valid, and reliable technique for measuring wound surface area. Skin Res Technol. 2016;22(4):443-50.

12. Hoogewerf CJ, Van Baar ME, Middelkoop E, Van Loey NE. Patient reported facial scar assessment: directions for the professional. Burns. 2013;40(2):347-53.
13. Goei H, Van Der Vlies CH, Tuinebreijer WE, Van Zuijlen P, Middelkoop E, Van Baar M. Predictive validity of short term scar quality on final burn scar outcome using the Patient and Observer Scar Assessment Scale in patients with minor to moderate burn severity. Burns. 2017;43(4):715-23.

14. Fischer TW, Peter WW. Direct and non-direct measurement techniques for analysis of skin surface topography. Ski Pharmacol Appl Ski Physiol. 1999;12:1-11.

15. Bloemen MCT, Van Gerven MS, Van Der Wal MBA, Verhaegen PDHM, Middelkoop E. An objective device for measuring surface roughness of skin and scars. J Am Acad Dermatol. 2010;64(4):706-15.

16. Trojahn C, Schario M, Dobos G, Kottner J. Reliability and validity of two in vivo measurements for skin surface topography in aged adults. Skin Res Technol. 2015;21:54-60.

17. Petit L, Bettoli DZV, Kang BDS, Martel P. Validation of 3D skin imaging for objective repeatable quantification of severity of atrophic acne scarring. Skin Res Technol. 2018;24(4):542-50.

18. Lumenta DB, Kitzinger H, Selig H. Objective quantification of subjective parameters in scars by use of a portable stereophotographic system. Ann Plast Surg. 2011;67(6):641-5.

19. Askaruly S, Ahn Y, Kim H, Vavilin A, Ban S, Kim PU, et al. Quantitative evaluation of skin surface roughness using optical coherence tomography in vivo. IEEE J Sel Top Quantum Electron. 2019;25(1):1-8.

20. Gankande TU, Duke JM, Danielsen PL, Dejong HM, Wood FM, Wallace HJ. Reliability of scar assessments performed with an integrated skin testing device - the DermaLab Combo. Burns. 2014;40(8):1521-9.

21. Lau JCM, Li-tsang CWP, Zheng YP. Application of tissue ultrasound palpation system (TUPS) in objective scar evaluation. Burns. 2005;31:445-52.

22. Nedelec B, Correa JA, Rachelska G, Armour A, LaSalle L. Quantitative measurement of hypertrophic scar: interrater reliability and concurrent validity. J Burn Care Res. 2008;29(3):501-11.

23. Simons M, Kee EG, Kimble R, Tyack Z. Ultrasound is a reproducible and valid tool for measuring scar height in children with burn scars: a cross-sectional study of the psychometric properties and utility of the ultrasound and 3D camera. Burns. 2017;43(5):993-1001.

24. Li JQ, Li-tsang CWP, Huang YP, Chen Y, Zheng YP. Detection of changes of scar thickness under mechanical loading using ultrasonic measurement. Burns. 2013;39:89-97.

25. Nedelec B, Correa JA, Rachelska G, Armour A, LaSalle L. Quantitative measurement of hypertrophic scar: intrarater reliability, sensitivity, and specificity. J Burn Care Res. 2008;29(3):489-500.

26. Agabalyan NA, Su S, Sinha S, Gabriel V. Comparison between high-frequency ultrasonography and histological assessment reveals weak correlation for measurements of scar tissue thickness. Burns. 2017;43(3):531-8.

27. Andrews CJ, Kempf M, Kimble R, Cuttle L. Skin thickness measurements increase with excision and biopsy processing procedures. Wound Repair Regen. 2017;25:338-40.

28. Tyack Z, Simons M, Kimble RM, Muller MJ, Leung K. The reproducibility and clinical utility of the $3 \mathrm{D}$ camera for measuring scar height, with a protocol for administration. Skin Res Technol. 2017;23(4):463-70.

29. Verhaegen PDHM, van Zuijlen PP, Pennings NM, van Marle J, Niessen FB, Van Der Horst CMAM, et al. Differences in collagen architecture between keloid, hypertrophic scar, normotrophic scar, and normal skin: an objective histopathological analysis. Wound Repair Regen. 2009;17:649-56.

30. Babalola O, Mamalis A, Lev-Tov H, Jagdeo J. Optical coherence tomography (OCT) of collagen in normal skin and skin fibrosis. Arch Dermatol Res. 2014;306(1):1-9. 
31. Gong P, Wood FM, Sampson DD, Mclaughlin RA. Imaging of skin birefringence for human scar assessment using polarizationsensitive optical coherence tomography aided by vascular masking assessment using polarization-sensitive optical. J Biomed Opt. 2014;19(12).

32. Jaspers MEH, Feroldi F, Vlig M, De Boer JF, Van Zuijlen PPM. In vivo polarization-sensitive optical coherence tomography of human burn scars: birefringence quantification and correspondence with histologically determined collagen density. J Biomed Opt. 2017;22(12).

33. Liew YM, Mclaughlin RA, Gong P, Wood FM, Sampson DD. In vivo assessment of human burn scars through automated quantification of vascularity using optical coherence tomography automated quantification of vascularity using. J Biomed Opt. 2013;18(6).

34. Es'haghian S, Gong P, Chin L, Harms K, Murray A, Rea S, et al. Investigation of optical attenuation imaging using optical coherence tomography for monitoring of scars undergoing fractional laser treatment. J Biophotonics. 2017;10:511-22.

35. Gong P, Es S, Harms K, Murray A, Rea S, Kennedy BF, et al. Optical coherence tomography for longitudinal monitoring of vasculature in scars treated with laser fractionation. J Biophotonics. 2016;9(6):626-36.

36. Gong P, Es'haghian S, Harms K-A, Murray A, Rea S, Wood $\mathrm{FM}$, et al. In vivo label-free lymphangiography of cutaneous lymphatic vessels in human burn scars using optical coherence tomography. Biomed Opt Express. 2016;7(12):1219-23.

37. Park KS, Choi WJ, Song S, Xu J, Wang RK. Multifunctional in vivo imaging for monitoring wound healing using sweptsource polarization-sensitive optical coherence tomography. Lasers Surg Med. 2017;50(3):213-21.

38. Es'haghian S, Kennedy KM, Gong P, Li Q, Chin L, Wijesinghe $\mathrm{P}$, et al. In vivo volumetric quantitative micro- elastography of human skin. Biomed Opt Express. 2017;8(5):121-8.

39. Es'haghian S, Kennedy KM, Gong P, Sampson DD, Mclaughlin RA, Kennedy BF. Optical palpation in vivo: imaging human skin lesions using mechanical contrast. J Biomed Opt. 2015; 20(1).

40. Shah RG, Devore D, Silver FH. Biomechanical analysis of decellularized dermis and skin: initial in vivo observations using OCT and vibrational analysis. J Biomed Mater Res Part A. 2018;106(5):1421-7.

41. Silver FH, Shah RG. Mechanical spectroscopy and imaging of skin components in vivo: assignment of the observed moduli. Skin Res Technol. 2019;25(1):47-53.

42. Reinholz M, Schwaiger H, Poetschke J, Epple A, Ruzicka T, Von Braunmuhl T, et al. Objective and subjective treatment evaluation of scars using optical coherence tomography, sonography, photography, and standardised questionnaires. Eur J Dermatol. 2016;26(December):599-608.

43. Ud-Din S, Foden P, Stocking K, Mazhari M, Al-Habba S, Baguneid $\mathbf{M}$, et al. Objective assessment of dermal fibrosis in cutaneous scarring: using optical coherence tomography, high frequency ultrasound and immuno-histo-morphometry of human skin. Br J Dermatol. 2019;6

44. Oliveira GV, Hawkins HK, Chinkes D, Burke A, Luiz A, Tavares $\mathrm{P}$, et al. Hypertrophic versus non hypertrophic scars compared by immunohistochemistry and laser confocal microscopy: type I and III collagens. Int Wound J. 2009;6(6):445-52.

45. Sideek MA, Teia A, Kopecki Z, Cowin AJ, Gibson MA. Co-localization of LTBP-2 with FGF-2 in fibrotic human keloid and hypertrophic scar. J Mol Histol. 2016;47(1).

46. Tey HL, Maddison B, Wang H, Ishiju Y, Mcmichaef A, Marks $\mathrm{M}$, et al. Cutaneous innervation and itch in keloids. Acta Derm Venereol. 2012;92(5):529-31.
47. Sasaki GH. Micro-needling depth penetration, presence of pigment particles, and fluorescein-stained platelets: clinical usage for aesthetic concerns. Aesthetic Surg J. 2017;37(1):71-83.

48. Van Zuijlen PPM, Ruurda JJB, Van Veen HA, Van Marle J, Van Trier AJM, Groenevelt F, et al. Collagen morphology in human skin and scar tissue: no adaptations in response to mechanical loading at joints. Burns. 2003;29:423-31.

49. Edwards SJ, Patalay R, Wakefield V, Karner C. Diagnostic accuracy of reflectance confocal microscopy using VivaScope for detecting and monitoring skin lesions: a systematic review. Clin Exp Dermatol. 2017;42(3):266-75.

50. Waddell A, Star P, Guitera P. Advances in the use of reflectance confocal microscopy in melanoma. Melanoma Manag. 2018;10(5).

51. Fabbrocini G, Ardigò M, Mordente I, Ayala F, Cacciapuoti S, Monfrecola G. Confocal microscopy images to monitor skin needling in the treatment of acne scars. J Clin Exp Dermatol Res. 2015;6(6).

52. Iftimia N, Ferguson RD, Mujat M, Patel AH, Zhang EZ, Fox W, et al. Combined reflectance confocal microscopy/optical coherence tomography imaging for skin burn assessment. Biomed Opt Express. 2013;4(5):178-87.

53. Hockberger PE, Skimina TA, Centonze VE, Lavin C, Chu S, Dadras S, et al. Activation of flavin-containing oxidases underlies light-induced production of $\mathrm{H} 2 \mathrm{O} 2$ in mammalian cells. Proc Natl Acad Sci U S A. 1999;96(May):6255-60.

54. Kobat D, Horton NG, Xu C. In vivo two-photon microscopy to 1.6-mm mouse cortex. J Biomed Opt. 2011;16(10).

55. Zoumi A, Yeh A, Tromberg BJ. Imaging cells and extracellular matrix in vivo by using second-harmonic generation and twophoton excited fluorescence. PNAS. 2002;99(17):11014-9.

56. Quinn KP, Leal EC, Tellechea A, Kafanas A, Auster ME, Veves A, et al. Diabetic wounds exhibit distinct microstructural and metabolic heterogeneity through label-free multiphoton microscopy. J Invest Dermatol. 2016;136(1):342-4.

57. Jones JD, Ramser HE, Woessner AE, Quinn KP. In vivo multiphoton microscopy detects longitudinal metabolic changes associated with delayed skin wound healing. Commun Biol. 2018;19(1):198.

58. Lentsch G, Balu M, Williams J, Lee S, Harris RM, König K, et al. In vivo multiphoton microscopy of melasma. Pigment Cell Melanoma Res. 2019;32(3):403-11.

59. Campagnola PJ, Millard AC, Terasaki M, Hoppe PE, Malone CJ, Mohler WA. Imaging of endogenous structural proteins in biological tissues. Biophys J. 2002;82(1):493-508.

60. Plotnikov SV, Millard AC, Campagnola PJ, Mohler WA. Characterization of the myosin-based source for secondharmonic generation from muscle sarcomeres. Biophys J. 2006;90(2):693-703.

61. Dombeck DA, Kasischke KA, Vishwasrao HD, Ingelsson M, Hyman BT, Webb WW. Uniform polarity microtubule assemblies imaged in native brain tissue by second-harmonic generation microscopy. PNAS. 2003;100(12):7087-6.

62. Bueno JM, Ávila FJ, Artal P. Second harmonic generation microscopy: a tool for quantitative analysis of tissues. In: Stanciu SG, editor. Microscopy and analysis. IntechOpen, 2016, pp. 99-220.

63. Huang C, Murphy GF, Akaishi S, Ogawa R. Keloids and hypertrophic scars: update and future directions. Plast Reconstr Surg Glob Open. 2013;1(25):1-7.

64. Cox G, Kable E, Jones A, Fraser I, Manconi F, Gorrell MD. 3-Dimensional imaging of collagen using second harmonic generation. J Strucutral Biol. 2006;141(2003):53-62.

65. Kottmann RM, Sharp J, Owens K, Salzman P, Xiao G, Phipps $\mathrm{RP}$, et al. Second harmonic generation microscopy reveals altered collagen microstructure in usual interstitial pneumonia versus healthy lung. Respir Res. 2015;16(61). 
66. Tilbury K, Lien C, Chen S, Campagnola PJ. Differentiation of col I and col III isoforms in stromal models of ovarian cancer by analysis of second harmonic generation polarization and emission directionality. Biophys J. 2014;106(2):354-65.

67. Liu Y, Zhu X, Huang Z, Cai J, Chen R, Xiong S, et al. Texture analysis of collagen second- harmonic generation images based on local difference local binary pattern and wavelets differentiates human skin abnormal scars from normal scars. J Biomed Opt. 2015;20(1):16021

68. Chen G, Liu Y, Zhu X, Huang Z, Cai J, Chen R. Phase and texture characterizations of scar collagen second-harmonic generation images varied with scar duration. Microsc Microanal. 2015;21(4):855-62.

69. Yildirim M, Quinn KP, Kobler JB, Zeitels SM, Georgakoudi I, Ben-Yakar A. Quantitative differentiation of normal and scarred tissues using second-harmonic generation microscopy. Scanning. 2016;38(6):684-93.

70. Mazumder N, Deka G, Wu W, Gogoi A, Zhuo G, Kao F. Polarization resolved second harmonic microscopy. Methods. 2017; 128:105-18.

71. Hristu R, Stanciu SG, Tranca DE, Stanciu GA. Improved quantification of collagen anisotropy with polarization-resolved second harmonic generation microscopy. $\mathrm{J}$ Biophotonics. 2017;10:1171-9.

72. Rosin NL, Agabalyan N, Olsen K, Martufi G, Gabriel V, Biernaskie J, et al. Collagen structural alterations contribute to stiffening of tissue after split-thickness skin grafting. Wound Repair Regen. 2016;24:263-74.

73. Meleshina AV, Rogovaya OS, Dudenkova VV, Sirotkina MA, Lukina MM, Bystrova AS, et al. Multimodal label-free imaging of living dermal equivalents including dermal papilla cells. Stem Cell Res Ther. 2018;9(84):1-12.

74. Torkian BA, Yeh AT, Engel R, Sun C, Tromberg BJ, Wong BJ. Modeling aberrant wound healing using tissue-engineered skin constructs and multiphoton microscopy. Arch Facial Plast Surg. 2004;6:180-7.

75. Qian HS, Weldon SM, Matera D, Lee C, Yang H, Fryer RM, et al. Quantification and comparison of anti- fibrotic therapies by polarized SRM and SHG- based morphometry in rat UUO model. PLoS One. 2016;11(6):1-13.

76. Weinigel M, Breunig HG, Uchugonova A, Konig K. Multipurpose nonlinear optical imaging system for in vivo and ex vivo multimodal histology in vivo and ex vivo multimodal histology. J Med Imaging. 2015;2(1):16003.
77. Rolopp A, Sákányi A, Aluszka D, Csati D, Vass L, Kolonics A, et al. Handheld nonlinear microscope system comprising a $2 \mathrm{MHz}$ repetition rate, mode- locked Yb-fiber laser for in vivo biomedical imaging. Biomed Opt Express. 2016;7(9):49-53.

78. Chung H-Y, Greinert R, Kartner FX, Chang G. Multimodal imaging platform for optical virtual skin biopsy enabled by a fiber-based two- color ultrafast laser source. Biomed Opt Express. 2019;10(2):514-25.

79. Debarre D, Beaurepaire E. Quantitative characterization of biological liquids for third-harmonic generation microscopy. Biophys J. 2007;92:603-312.

80. Witte S, Negrean A, Lodder JC, De Kock CPJ, Testa Silva G, Mansvelder HD, et al. Label-free live brain imaging and targeted patching with third-harmonic generation microscopy. Proc Natl Acad Sci U S A. 2011;108(15):5970-5.

81. Weigelin B, Bakker G, Friedl P. Principles of interface guidance and microvesicle dynamics Intravital third harmonic generation microscopy of collective melanoma cell invasion. Intra Vital. 2012;1(1):32-43.

82. Tsai M, Cheng Y, Chen J, Liao Y, Sun C. Differential diagnosis of nonmelanoma pigmented skin lesions based on harmonic generation microscopy. J Biomed Opt. 2014;19(3).

83. Yu C, Tai S, Kung C, Wang I, Yu H, Lee W, et al. In vivo and ex vivo imaging of intra-tissue elastic fibers using thirdharmonic-generation microscopy. Opt Express. 2007;15(18): 527-32.

84. Chen S, Wu H, Sun C. In vivo harmonic generation biopsy of human skin. JBO Lett. 2009;14(6):25-7.

85. Osseiran S, Dela Cruz J, Jeong S, Wang H, Fthenakis C, Evans CL. Characterizing the stratum corneum structure, barrier function, and chemical content of human skin with coherent Raman scattering imaging. Biomed Opt Express. 2018;9(12).

86. Foltynski P. Ways to increase precision and accuracy of wound area measurement using smart devices: advanced app Planimator. PLoS One. 2018;13(3):1-16.

87. Van Huizen L, Kuzmin N, Barbe E, Van der Velde S, Te Velde E, Groot M. Second and third harmonic generation microscopy visualizes key structural components in fresh unprocessed healthy human breast tissue. J Biophotonics. 2019;12(6):e201800297.

88. Schindelin J, Arganda-carreras I, Frise E, Kaynig V, Longair M, Pietzsch T, et al. Fiji: an open-source platform for biologicalimage analysis. Nat Methods. 2012;9(7):676-82.

Open Access This chapter is licensed under the terms of the Creative Commons Attribution 4.0 International License (http://creativecommons. org/licenses/by/4.0/), which permits use, sharing, adaptation, distribution and reproduction in any medium or format, as long as you give appropriate credit to the original author(s) and the source, provide a link to the Creative Commons license and indicate if changes were made.

The images or other third party material in this chapter are included in the chapter's Creative Commons license, unless indicated otherwise in a credit line to the material. If material is not included in the chapter's Creative Commons license and your intended use is not permitted by statutory regulation or exceeds the permitted use, you will need to obtain permission directly from the copyright holder.

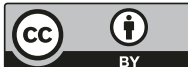

